\title{
Energy-Efficient Biometrics-Based Remote User Authentication for Mobile Multimedia I OT Application
}

\author{
Sungju Lee, Jaewon Sa, Hyeonjoong Cho*, and Daihee Park \\ Dept. of Computer Convergence Software, \\ Korea University, Sejong, South Korea \\ [e-mail: peacfeel, sjwon92, raycho, dhpark@korea.ac.kr] \\ *Corresponding author: Hyeonjoong Cho
}

Received September 2, 2016; revised November 17, 2016; revised January 13, 2017; accepted August 14, 2017; published December 31, 2017

\begin{abstract}
Recently, the biometric-based authentication systems such as FIDO (Fast Identity Online) are increased in mobile computing environments. The biometric-based authentication systems are performed on the mobile devices with the battery, the improving energy efficiency is important issue. In the case, the size of images (i.e., face, fingerprint, iris, and etc.) affects both recognition accuracy and energy consumption, and hence the tradeoff analysis between the both recognition accuracy and energy consumption is necessary. In this paper, we propose an energy-efficient way to authenticate based on biometric information with tradeoff analysis between the both recognition accuracy and energy consumption in multimedia IoT (Internet of Things) transmission environments. We select the facial information among biometric information, and especially consider the multicore-based mobile devices. Based on our experimental results, we prove that the proposed approach can enhance the energy efficiency of $G A B O R+L B P+G R A Y V A L U E, G A B O R+L B P, G A B O R$, and $L B P$ by factors of $6.8,3.6$, 3.6, and 2.4 over the baseline, respectively, while satisfying user's face recognition accuracy.
\end{abstract}

Keywords: Multicore-based handheld device, energy consumption, biometric recognition, image compression, image transmission, tradeoff analysis 


\section{Introduction}

Recently, the biometric-based authentication systems such as FIDO (Fast Identity Online) are increased in mobile computing environments [1]. In traditional biometric recognition, the system consists of enrollment and identification; biometric information is stored remotely in a central database, and the identification device communicates with the database through a wired network [2]. Recently, some researchers have sought to integrate biometric recognition systems into wireless networks [3-5]. The biometric-based authentication systems are performed on the mobile devices with the battery, the improving energy efficiency is important issue.

When transmitting image data from handheld devices through a wireless network, we need to minimize energy consumption while still providing satisfactory accuracy for biometric recognition. Although compressed image can reduce the transmission energy consumption, the cost associated with the encoding computation may not be negligible. Furthermore, image compression techniques such as JPEG [6-9] may degrade the quality of the original image. This quality degradation reduces the accuracy of biometric recognition.

Recently, the development of high-performance, multicore handheld devices was announced. High-performance, multicore handheld devices will also be used as VSN (Video Sensor Network) processing nodes in the near future. Traditionally, parallel processing has been broadly used to decrease the execution time of an application. With the advances in multicore technology, multiprocessing techniques at a system software level can reduce the energy consumption [10-14]. However, the total power consumption may be increased with parallel processing on multicore processors because there are more physical cores [14].

When a given multimedia IoT transmission environment (i.e., compression application parallelism, network bandwidth, multicore-based handheld device) is specified, we need an energy-efficient method to transmit the biometric information from the handheld device and satisfy the user's requirement for biometric recognition accuracy. To efficiently transmit the compressed biometric image with limited handheld resources and maintain acceptable biometric recognition accuracy, we use a performance metric including collective performance. Note that, this performance metric is measured only once to find out characteristics of the energy consumption and accuracy according to given multicore-based handheld device and face recognition method. After measurement of performance metric, we can apply proposed approach for many combination of multicore-based handheld device and face recognition method without any experimental test. Our main goal is to improve the energy efficiency of the multicore-based handheld device and satisfy the required biometric recognition accuracy by implementing optimal parameters (i.e., CPU frequency, number of cores, and compression rate) in the given multimedia IoT transmission environment. First, the tradeoff between energy consumption and biometric recognition accuracy is analyzed with E-A (i.e., Energy-Accuracy) analysis. Note that, the energy consumption consists of both compression and transmission energy.

To improve the compression energy efficiency, we optimize the machine's parameters (i.e., CPU frequency, number of cores) of the multicore-based handheld devices according to compression application parallelism. Also, to improve the transmission energy efficiency, we optimize the compression rate. For example, when the compression rate increase (i.e., compression parameters increases), transmission energy consumption and biometric 
recognition accuracy decrease. With the optimal parameters of multicore-based handheld devices and compression applications, the biometric image can be energy-efficiently compressed and transmitted with acceptable biometric recognition accuracy. In addition, we consider the un_compression scenario to reduce energy consumption according to the wireless network bandwidth, and can efficiently transmit the biometric image under the limited handheld resources and acceptable biometric recognition accuracy by using $E-A$ analysis.

The face recognition has certain advantages such as being natural and passive as opposed to other biometric techniques demanding cooperative subjects, such as fingerprint or iris recognition. It has been widely used in access control, identification systems, and surveillance. In these reasons, we select the facial information among biometric information and consider the multicore-based mobile devices. To the best of our knowledge, this is the first report for improving the energy efficiency of a multicore-based handheld device and still satisfying the required biometric recognition accuracy by optimizing the parameters (i.e., CPU's frequency, number of cores, and compression rate) of the given VSN transmission environment.

In our experiments, we used commercial multicore processors based on Intel quad-core i7 for analyzing the device's characteristics, and we analyzed energy efficiency by evaluating the actual power consumption with a WT210 tool [15]. We also used compression algorithm JPEG [6], Yale face data [16], and diverse network conditions (i.e., network bandwidth). Based on our experimental results with $E$ - $A$ analysis, the proposed method can improve the energy efficiency of $G A B O R+L B P+G R A Y$ VALUE, GABOR+LBP, GABOR, and $L B P$ by factors of 6.8, 3.6, 3.6, and 2.4 over the baseline scenario (i.e., default parameter settings), respectively, and retain acceptable face recognition accuracy.

The rest of the paper is structured as follows. Section 2 describes previous quality-optimization approaches, a relevant handheld device based on multicore processors, and the energy consumption required by the compression application's parallelism. Section 3 explains the proposed approach for $E$ - $A$ analysis and parameter optimization. Finally, Sections 4 and 5 describe the experimental results and our conclusions, respectively.

\section{Related Work}

\subsection{Quality-Optimization Approaches}

Image compression techniques such as JPEG [7-9] may degrade the quality of the original image. To improve the face recognition accuracy, we can consider the high quality with decreased compression rate. However, energy and execution time more consumed for high quality during compressed/transmitted face image on handheld devices. To improve energy efficiency without compensating quality, compression techniques may be modified, or collective performance (i.e., power consumption, execution time, and quality) could be optimized by changing major parameters (i.e., compression rate and frame interval). To optimize the collective performance, $p$-r $-d$ (i.e., power, rate, and distortion) analysis have been reported by $[17,18]$. These studies analyzed the relation of $p-r-d$, and developed an optimization approach to improve energy efficiency for handheld devices. [19] et al. proposed an energy-efficient JPEG compression technique for wireless handheld devices that uses fast DCT computation to increase efficiency and maintain acceptable image quality. These works did not consider multicore-based handheld devices, but they did consider Image quality degradation according to compression rate.

Image quality degradation affects face recognition accuracy [3-5]. These studies analyzed the effect of degradation due to image compression on face recognition accuracy. Generally, 
high-quality images can provide high face recognition accuracy, but they require more energy consumption. The image quality and computational characteristics depend on the type of compression techniques being used, such as JPEG or JPEG2000 [5-9]. Further, energy consumption depends on the compression application's parallelism and the specifications of the multicore-based handheld device. Therefore, we need a collective analysis to determine how to increase energy efficiency on the multicore-based handheld device and maintain acceptable face recognition accuracy.

\subsection{Handheld Device based on Multicore Processors}

Improving the CPU's frequency is no more reasonable because of the high power consumption and heat dissipation. Using multicore processors is one of solution to resolve this issue [10]. Also recently, handheld devices such as smart phone have several cores for high performance computing. According to the hardware architecture model, commercial multicore processors have different characteristics. For instance, in Intel's multicore architecture, two cores share the L2 cache. In one of the chipmaker's multicore architecture, each core allocates the L2 cache. In the commercial computer system, the various computer systems can be configured with the hardware components (i.e., memory, hard disk, IO devices, etc.). Since the power consumption and execution time of the commercial multicore processor depend on the hardware architecture model, it is hard to optimize the power consumption and execution time parameters $[13,14]$.

\subsection{Energy Compression by Application Parallelism}

Advanced technologies and design trends strongly indicate that multicore processors will be able to exploit Dynamic Voltage and Frequency Scaling (DVFS or DVS) [10-14]. The execution time of an application on these machines (i.e., multicore platforms with DVFS or DVS) depends on not only the commercial multicore platforms but also application's parallelism. Amdahl's law can forecast the speedup of parallel processing through both the sequential portion of an application and the number of processors used, and thus, to predict the power consumption, several researches exploited the idea of Amdahl's law [13].

\section{Proposed Approach}

To efficiently transmit a compressed face image under the limited handheld resources and retain acceptable face recognition accuracy, we use a performance metric including collective performance. For example, a user requires the minimum energy consumption possible to carry out a process which will satisfy the required face recognition accuracy under the given USN transmission conditions (i.e., compression application parallelism, network bandwidth, multicore-based handheld device). To solve the problem, we first analyze the collective performance (i.e., face recognition accuracy and energy consumption), and then determine the optimal combination of machine and compression application parameters (i.e., CPU frequency $f$, number of cores $n$, and compression parameter q). Fig. 1 shows the overall proposed approach.

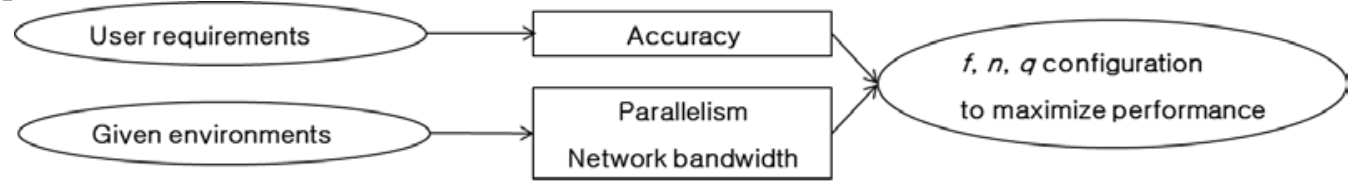

Fig. 1, Proposed approach with optimal parameters 
To improve collective performance, we first analyze the face recognition accuracy and energy consumption (i.e., the compression and transmission) with each parameter. Note that, in this paper, we define the given parameters (i.e., compression parallelism $p$, network bandwidth $b$ ) as those that the user does not determine, as opposed to control parameters (i.e., CPU frequency $f$, number of cores $n$, and compression parameter $q$ ). Note that, we use a performance metric with the power consumption of a test compression application measured only once with each combination of the machine's power consumption and the face recognition ratio by CPU frequency and given number of cores. Note that, this performance metric is measured only once to find out characteristics of the energy consumption and accuracy according to given multicore-based handheld device and face recognition method. After measurement of performance metric, we can apply proposed approach for many combination of multicore-based handheld device and face recognition method without any experimental test. Finally, we find the optimal parameters for energy efficiency in transmitting face image data without degrading the accuracy of face recognition by using greedy approach.

\subsection{E-A analysis}

\subsubsection{Face recognition accuracy}

In general, image compression techniques such as JPEG are most commonly used for lossy compression. Consequently, the image quality is degraded versus the original image, which affects the accuracy of face recognition. In compression techniques such as JPEG and JPEG2000, the user can control the image quality with the compression parameter q. Fig. 2 shows a sample face image that demonstrates the effect of the compression parameter q setting. To provide the highest face recognition accuracy, $q$ should be maximized (i.e., $q=100$ ). Although face recognition accuracy increases with higher $q$, more energy is required to carry out compression and transmission.
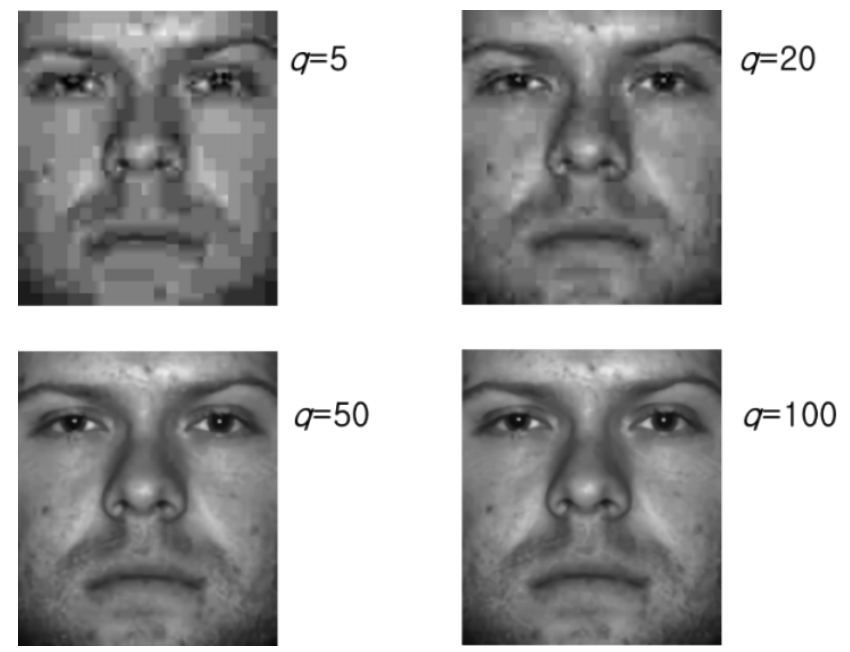

Fig. 2. Face image with the compression parameters $q$

To maintain the user's required face recognition accuracy with the compression parameter $q$, we represent the face recognition accuracy as equation (1). We then obtain the compression parameter $q$ with the inverse function of $A$ as shown in equation (2). 


$$
\begin{aligned}
& A(q)=\text { Face Recognition Accuracy } \\
& q=A^{-1}(\text { Face Recognition Accuracy) }
\end{aligned}
$$

\subsubsection{Compression energy}

To calculate the parallel compression energy consumption, we exploit the idea of [11]. The power consumption varies during the execution of a given compression application. Accordingly, the power consumption and sequential execution time are required to measure at least once for analyzing the characteristics of a machine. Therefore, the power consumption of compression program on given commercial multicore platform can be predicted with idea of Amdahl's law and performance metrics.

To conduct the power performance metrics, the power consumption was measured at some points and calculated the average. For simplicity, this average value was used as the power consumption value. Note that a compression application is composed of a sequential portion (having several data dependency) and a parallel portion (no having any data dependency). We describe the power consumption of the sequential portion of the compression application with 1 core as $W_{\text {sequential }}(f, 1)$ and the power consumption of the parallel portion of the compression application with $n$ cores as $W_{\text {parallel }}(f, n)$. Also, the total execution time $T\left(f, n, p_{\text {app }}\right)$ with diverse numbers of cores is able to be estimated by using Amdahl's law. It should also be noted that the execution time depends on the compression parameter $q$. Therefore, we represent the compression energy on a multicore-based handheld device as equation (3).

$$
E_{\text {comp }}(p, f, n, q)=W_{\text {sequential }}(f, 1) \times T_{\text {comp }}(f, q) \times(1-p)+W_{\text {parallel }}(f, n) \times T_{\text {comp }}(f, q) / n \times(p)
$$

\subsubsection{Transmission energy}

The network's condition and compression rate affect transmission energy consumption. Therefore, the transmission energy consumption is described as equation (4). $M$ is the compressed data size decided by the compression parameter (i.e., q), and $b$ is the network bandwidth (i.e., unit: MB per second).

$$
E_{\text {trans }}(q, b)=W_{\text {trans }} \times M(q) / b
$$

Finally, when the transmission environments (i.e., compression application parallelism, network bandwidth, multicore-based handheld device) are given, the energy consumption to compress/transmit the face image by the parameters (i.e., $p, b, f, n, q$ ), and $E$ is represented as equation (5).

$$
E(p, b, f, n, q)=E_{\text {comp }}(p, f, n, q)+E_{\text {trans }}(q, b)
$$

\subsubsection{Collective performance}

To improve the collective performance of face recognition accuracy and energy efficiency, we express the collective performance as equation (6). Note that the compression/transmission energy consumption and face recognition accuracy are normalized based on the maximum CPU frequency, the number of cores, and default $q(=50)$. The compression parameter $q$ 
affects not only face recognition accuracy, but also energy consumption. In fact, face recognition accuracy depends on the type of compression and recognition approaches. Thus, the level of accuracy associated with each type of face recognition approach should be measured with compression parameter $q$ at least once. With these measured and computed results, we can construct an experiment-based model that minimizes the energy consumption and still satisfies the required face recognition accuracy by using optimal parameters.

$$
P(p, b, f, n, q)=A(q) / E(p, b, f, n, q)
$$

\subsection{E-A optimization}

To efficiently transmit the face image, we need a method to optimize the control parameters (i.e., $f, n, q$ ) with respect to the given parameters (i.e., $b, p$ ). Fig. 3 shows examples of the collective performance $P(=A / E)$ with improved control parameters and the given parameters (i.e., $0.1 \mathrm{MB} / \mathrm{s}, p=0.9$ and $10 \mathrm{MB} / \mathrm{s}, p=0.7$ ). In Fig. 3, we discover that the optimal control parameters with regard to the given parameters should be found in the large search space. In the low-bandwidth network, the transmission energy has a stronger effect on $P$ than the compression energy, and thus, $P$ is sensitive to the effect of $q$. Therefore, transmission energy consumption is increased on the low-bandwidth network (i.e., $0.1 \mathrm{MB} / \mathrm{s}$, $p=0.9$ ), and $P$ tends to increase in response to low $q$, high $f$, and $n$ as shown in Fig. 3 (a). Although low $q$ can increase $P$, the accuracy of face recognition accuracy may not satisfy the user's requirement. To solve the problem, we select $q$ to satisfy the user's required face recognition accuracy by using equation (2). In contrast, on the high-bandwidth network (i.e., $10 \mathrm{MB} / \mathrm{s}, p=0.7$ ) as shown in Fig. 3 (b), the compression energy has more effect on $P$ than the transmission energy. Because the compression energy significantly depends on the parallelism $p$, the parameters of CPU frequency $f$ and number of cores $n$ have a greater effect on $P$ than $q$. Note that, to understand the device's characteristics, the power consumption and execution time are required to measure at least once. In addition, $p$ should be analyzed for the given compression application such as JPEG.

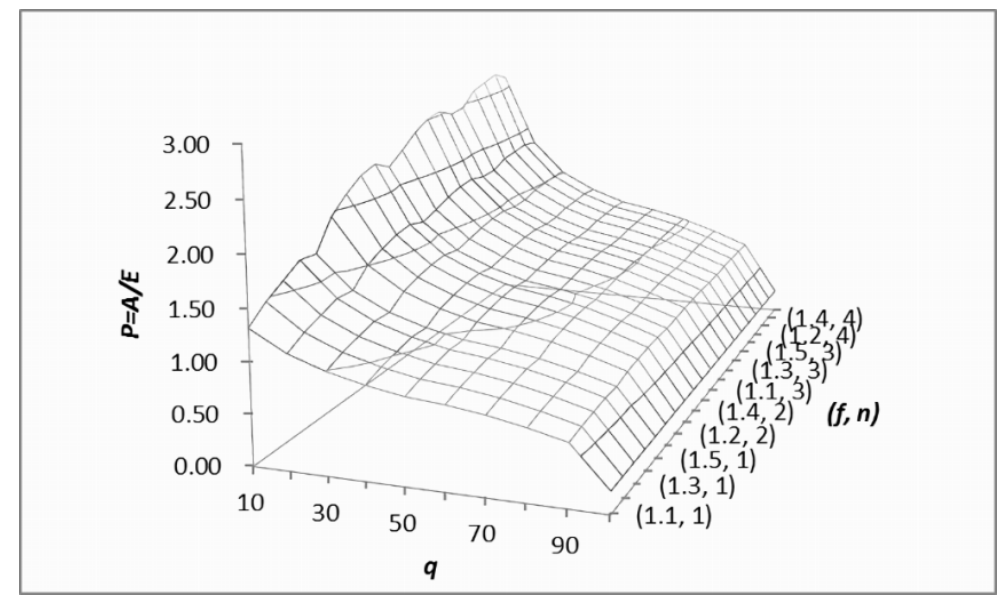

(a) $0.1 \mathrm{MB} / \mathrm{sec}, p=0.9$ 


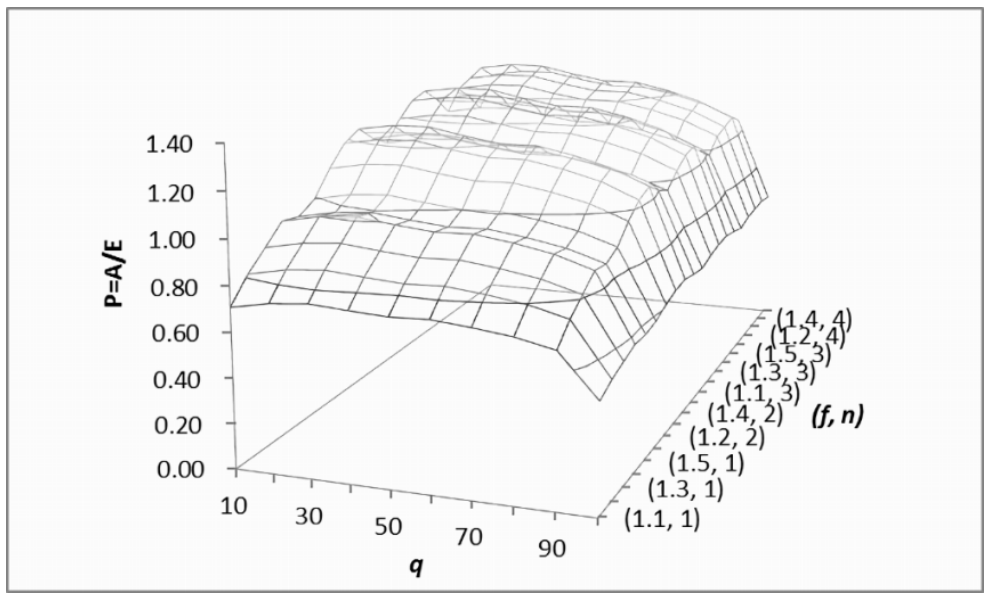

(b) $10 \mathrm{MB} / \mathrm{sec}, p=0.7$

Fig. 3. Examples of collective performance $P(=A / E)$ with the control parameters $(f, n, q)$ and given parameters $(p, b)$

\subsubsection{Compressed and un_compressed face images}

In addition, we consider the un_compression scenario to reduce energy consumption according to the wireless network bandwidth. Although the compression scenario can reduce the transmission data size, a greater total energy consumption (i.e., the compression and the transmission energy) may be required due to the compression energy consumed by the compression operation itself. Because the transmission time of the high-network bandwidth is less than that of the low-network bandwidth, more transmission energy may be required for the compression scenario than for the un_compression scenario. Consequently, we should determine which transmission scenario, compression or un_compression, is optimal. Finally, we can efficiently transmit the biometric image under the limited handheld resources and acceptable face recognition accuracy by using $E$ - $A$ analysis.

In some cases, the compression techniques consume more energy than was saved in comparison with the un_compression scenario. In such cases, because the original face image (i.e., un_compression) provides better face recognition accuracy, we should transmit the face image without any compression. Therefore, the compressed and the un_compressed approaches should be compared, and we must determine which scenario is optimal by carrying out equation (7).

$$
D(p, b, f, n, q)=P(p, b, f, n, q)-P(b)
$$

\subsubsection{Optimization parameters}

In this section, a greedy approach to optimize control parameters (i.e., $f, n, q)$ is proposed for energy efficiency in transmitting face image and still satisfying the user's requirement for face recognition accuracy. Algorithm 1 shows a method to minimize energy consumption by taking advantage of the optimal control parameters. Note that, because the optimal compression and transmission energy consumption are computed after finding optimal control parameters, we select either compression or un_compression at Step 4. 
Algorithm 1. Minimization of energy consumption with optimal parameters

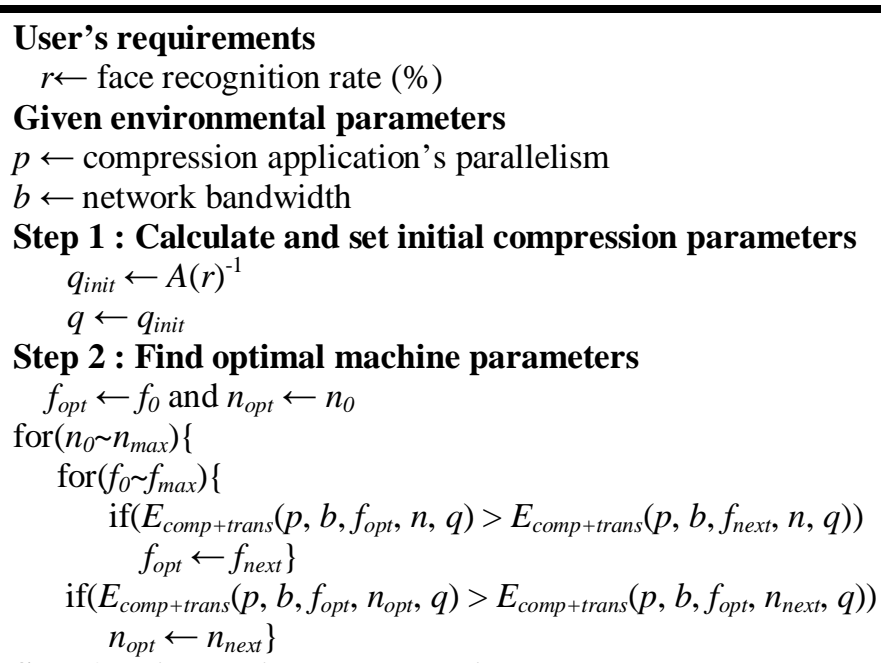

Step 3 : Find optimal compression parameters

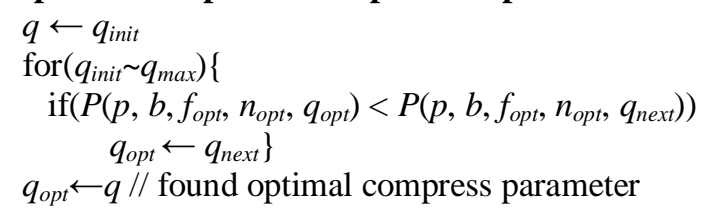

Step 4: Select face image transmission by compression or un_compression $\operatorname{If}(D(p, b, f, n, q)>0)$ compression and transmission Else un_compression and transmission

\section{Experimental Environments}

In this section, we present our experimental results. The experimental environment is described in Section 4.1. Afterward, the energy efficiency caused by using the $E-A$ analysis is explained in Section 4.2.

\subsection{Experimental environments}

\subsubsection{Remote face recognition system}

We assume that the handheld device captures a face image, compresses it, and finally transmits it with ID through a Network Interface Card (NIC). We used JPEG to compress face data drawn from the Yale dataset [16]. For our parallel compression algorithm, we parallelized JPEG [13,14,20] using pthreads [21].

To recognize the face, serveral techniques such as LBP (Local Binary Patterns), LDA (Linear Discriminant Analysis), GABOR, and GRAY VALUE can be used [22-27]. In the technizues, we use the LBP [24] and SVDD (Support Vector Data Description) [25] approach for face feature extraction and learning/test, respectively. LBP is computationally efficient, and the method is highly effective for face recognition. Nonetheless, the approaches depicted in this paper can be readily applied to work with alternative local descriptors. In LBP-based approaches, the face image is divided into a grid of small, non-overlapping regions, where a histogram of the LBP for each region is constructed. The similarity of the two images can be 
calculated by summing the similarity of histograms from the corresponding regions. The SVDD approach analyzes the data by searching for a minimum size of super-spheres containing as many points of one class of data as possible. Note that the SVDD can differentiate between the two classes by learning only one class. The overall remote face recognition process is shown by Fig. 4, which shows the enrollment verification processes of the face recognition system. In addition, we use GABOR+LBP+GRAY VALUE [22], $G A B O R+L B P$ [22], GABOR [23], LBP [24], and GRAY VALUE to compare the performance according to face feature extraction techniques.

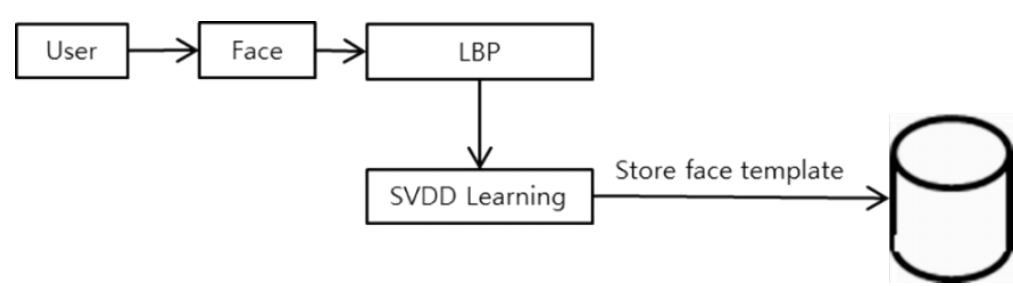

(a) Enrollment process

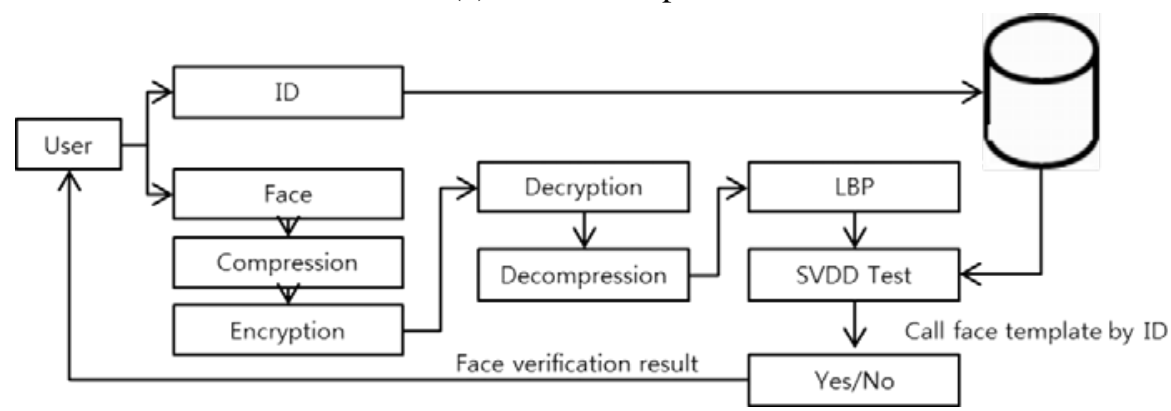

(b) Remote recognition process

Fig. 4. Face enrollment and remote recognition process

\subsubsection{Multicore-based handheld device environments}

To evaluate the change in energy efficiency that results from using the $E-A$ analysis, we configured the experimental environment as follows. We used a multicore-based handheld device (i.e., Intel quad-core i7-based notebook). As shown in Table 1, we configured the wireless network conditions from $0.1 \mathrm{MB} / \mathrm{s}$ to $10 \mathrm{MB} / \mathrm{s}$.

Table 1. Experimental configuration environments

\begin{tabular}{|l|l|}
\hline Processor & Intel i7 720QM \\
\hline Frequency range & $1.0 \mathrm{GHz}-1.5 \mathrm{GHz}$ \\
\hline Frequency step & $133 \mathrm{MHz}$ \\
\hline Maximum number of cores & 4 \\
\hline Network device Wireless & Intel Centrino(R) Advanced-N AGN \\
\hline
\end{tabular}


To measure the actual power consumption, we used a WT210 power meter [14]. The power consumption of the entire system was considered for the compression/transmission step with various network bandwidths. Table 2 shows the power consumption with various CPU frequencies and numbers of cores. In addition, the network card consumed 29 Watt.

Table 2. Power consumption on 4-core handheld device (Watt)

\begin{tabular}{|c|c|c|c|c|}
\hline & 1 core & 2 cores & 3 cores & 4 cores \\
\hline $1064 \mathrm{MHz}$ & 28.5 & 31.0 & 34.0 & 37.5 \\
\hline $1197 \mathrm{MHz}$ & 29.0 & 32.5 & 36.5 & 40.0 \\
\hline $1329 \mathrm{MHz}$ & 29.5 & 33.5 & 38.5 & 43.5 \\
\hline $1462 \mathrm{MHz}$ & 30.0 & 36.0 & 42.0 & 47.0 \\
\hline $1595 \mathrm{MHz}$ & 33.0 & 41.5 & 48.0 & 53.5 \\
\hline
\end{tabular}

\subsection{Results of $P(=A I E)$}

To evaluate the energy efficiency that results from using the $E$ - $A$ analysis, we compared baseline scenario against the proposed approach. The baseline scenario is that the machine's and compression's parameters are set to their respective defaults (i.e., CPU frequency is 1595 $\mathrm{MHz}$, the number of cores is one, and $q$ is 50 ). In our proposed approach, the machine's and compression's parameters are optimized. In the proposed approach, the ideal machine and compression parameters are collectively set by using the $E-A$ analysis. Note that, we used JPEG to compress the face image, and the parallelism is 0.96. Additionally, we assume that the user requires the face recognition rate to be higher than 0.9 (i.e., $p=0.96$ and $A(q)>0.9$ ).

We first confirmed that the proposed approach can reduce the total energy consumption (i.e., compression and transmission) with different network bandwidths. The transmission energy consumption depends on the network bandwidth $b$, and compression parameter $q$. In our experimental environments, with increased bandwidth $b$ (i.e., from $0.1 \mathrm{MB} / \mathrm{s}$ to $10 \mathrm{MB} / \mathrm{s}$ ), because the influence of transmission energy by $q$ was decreased, the reduction of compression energy used by the optimal parameters $f, n, q$ can effectively reduce the total energy consumption (i.e., by factors of 1.4 and 2.6, respectively). Note that, in the high-bandwidth network (i.e., $b>2.5 \mathrm{MB} / \mathrm{s}$ ), the un_compression approach can reduce the total energy consumption more than either the baseline scenario or the compression approach by optimal parameters as shown in Fig. $\mathbf{5}$. 


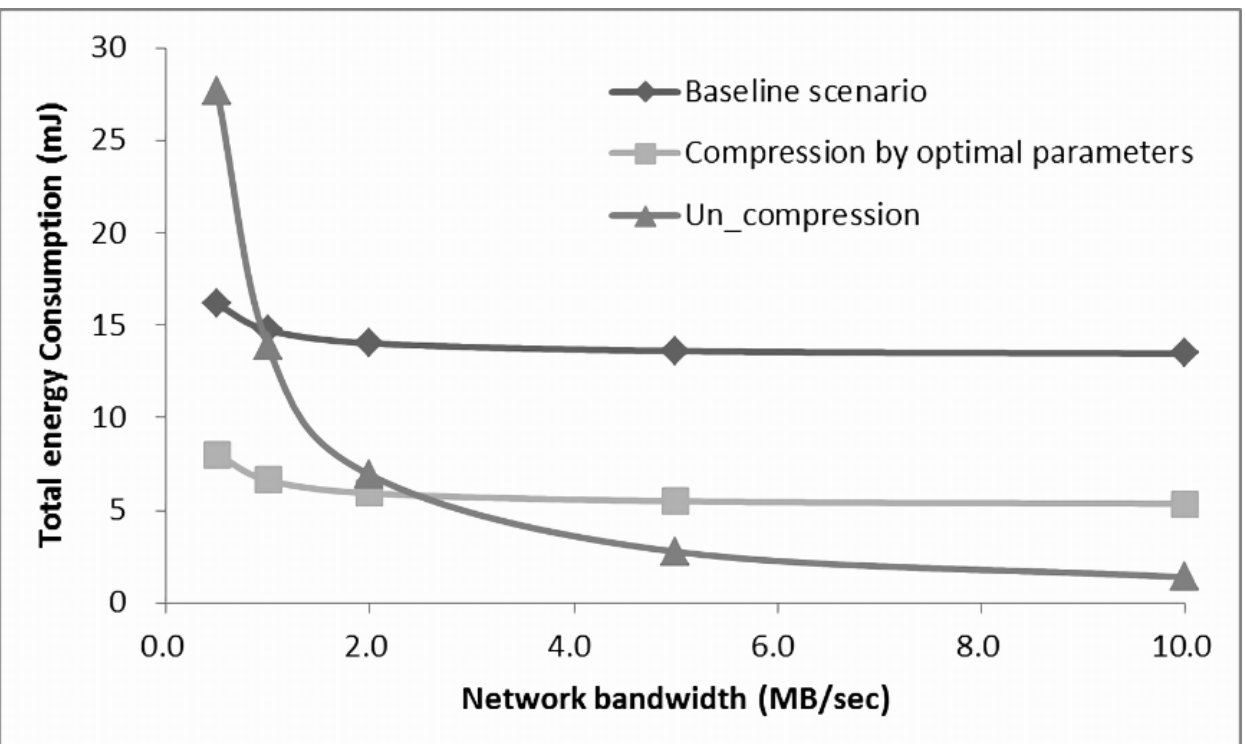

Fig. 5. Total energy consumption with various network bandwidths

We confirmed that the proposed approach can improve $P$ with various network bandwidths. Fig. 6 shows the comparison of performance between the baseline and proposed approaches on the low (i.e., $b=0.1 \mathrm{MB} / \mathrm{s}$ ), moderate (i.e., $b=1.0 \mathrm{MB} / \mathrm{sec}$ ), and high (i.e., $b=10.0$ $\mathrm{MB} / \mathrm{sec}$ )-bandwidth networks. On various network bandwidths, the proposed approach can reduce the total energy consumption by factors of 1.4, 2.1, and 2.6 over the baseline, respectively, and still satisfies the user's specified requirement for face recognition accuracy (i.e., $A(q)>0.9)$.

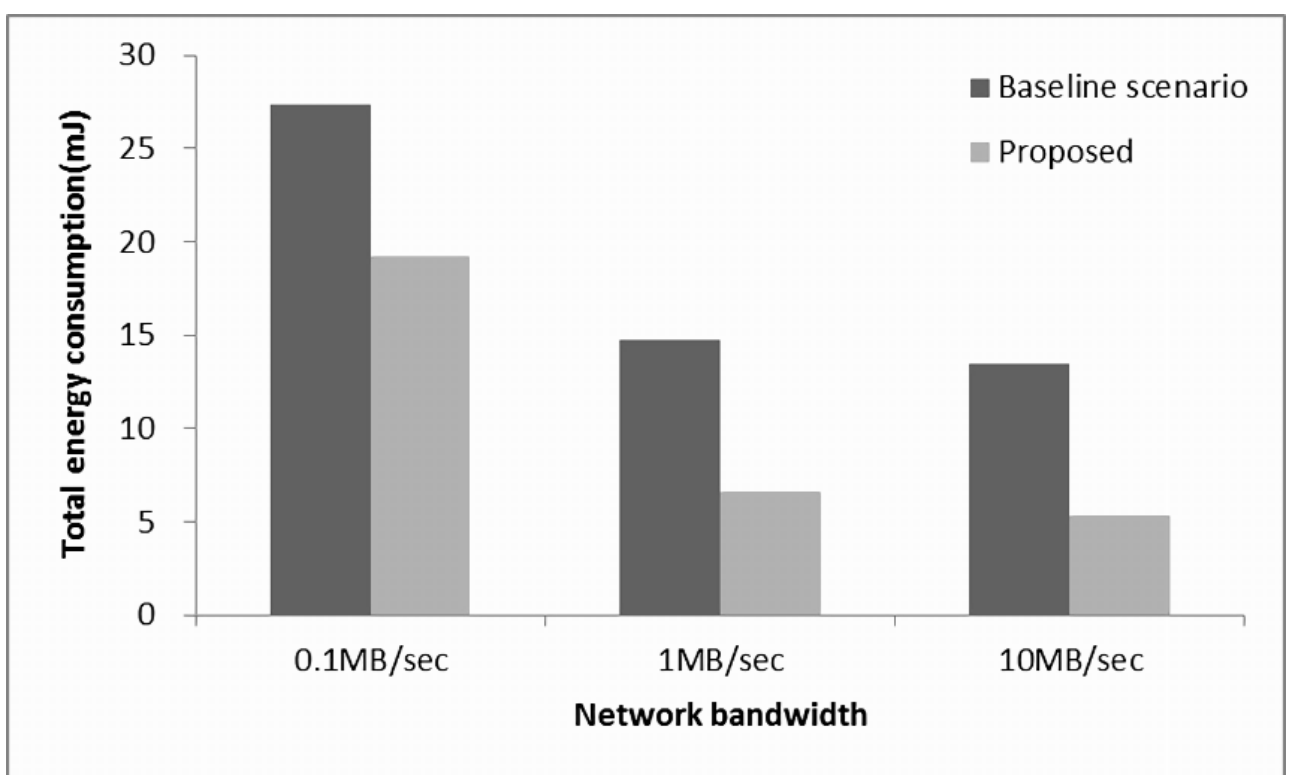

(a) Total energy consumption 


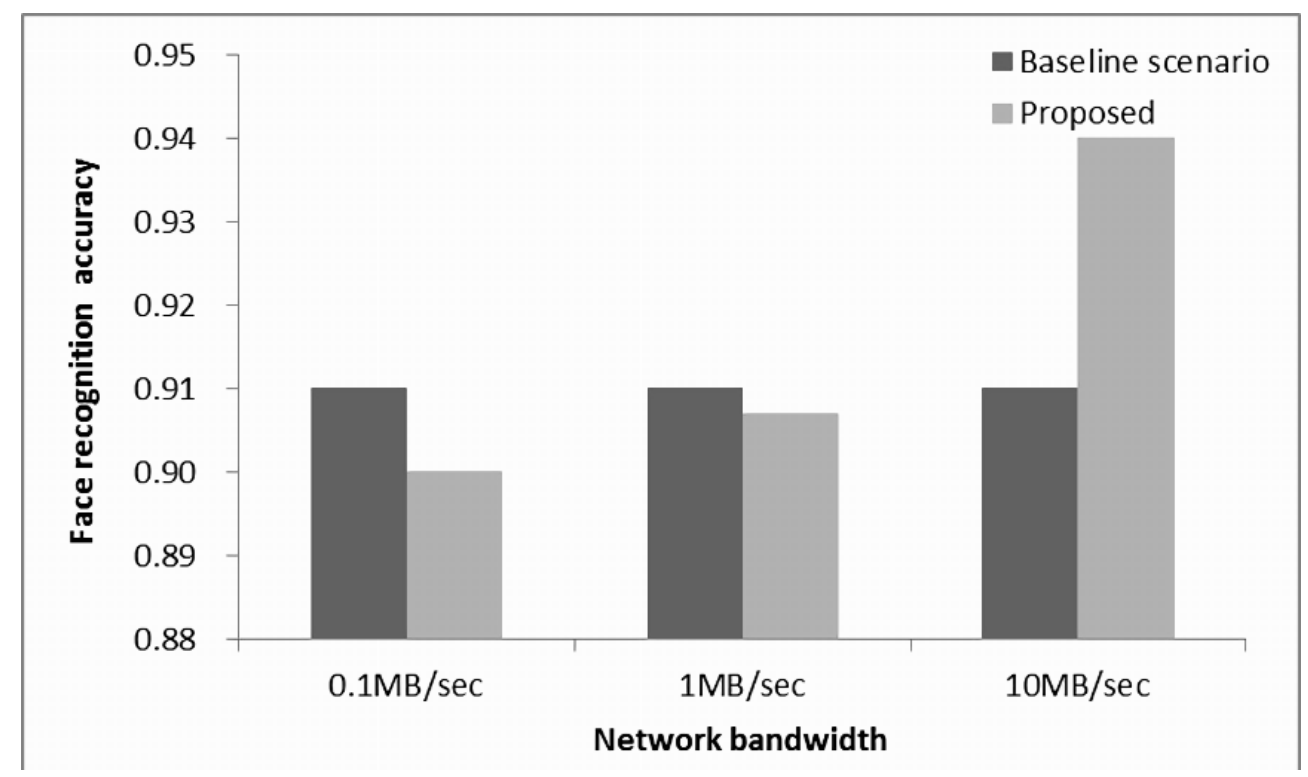

(b) Face recognition accuracy

Fig. 6. Comparison of performance between baseline and proposed approaches on various network bandwidths

Finally, we confirmed that the proposed approach can reduce the energy consumption while the user's specified requirement for face recognition accuracy with various various face feature extraction techniques. To meause the energy consumption and face recognition, we used the five face feature extraction techniques with GABOR+LBP+GRAY VALUE, $G A B O R+L B P, G A B O R, L B P$, and GRAY VALUE. Fig. 7 shows the comparison of performance between the baseline and proposed approaches on five techniques. In the Fig. 7 (b), on various techniques excpt GRAY VALUE, the proposed approach can reduce the total energy consumption by factors of 6.8, 3.6, 3.6, and 2.4 over the baseline, respectively. Since the face recognition performance of GRAY VALUE is low, large amount of energy is consumed in order to stisfy the user's face recognition accuracy. Fig. 7 (b) shows the comparison of face recognition accuracy between the baseline and proposed approaches on five techniques. On various techniques, although the face recognition accuracies were degreaded compared to baseline scenario, still satisfies the user's specified requirement for face recognition accuracy (i.e., $A(q)>0.9$ ). Note that, in the GRAY VALUE, baleline scenaio did not guarentee the user's face recognition accuracy. In the contrast, proposed approach can gurarentee the user's face recognition accuracy. 


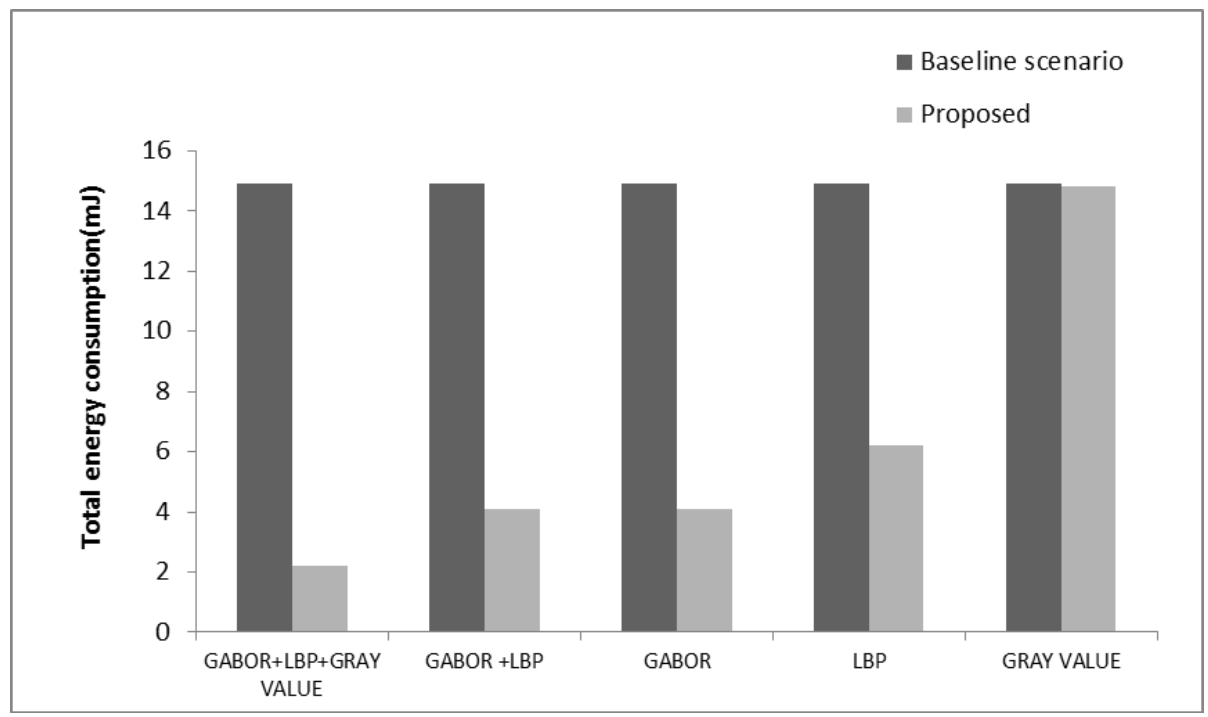

(a) Total energy consumption

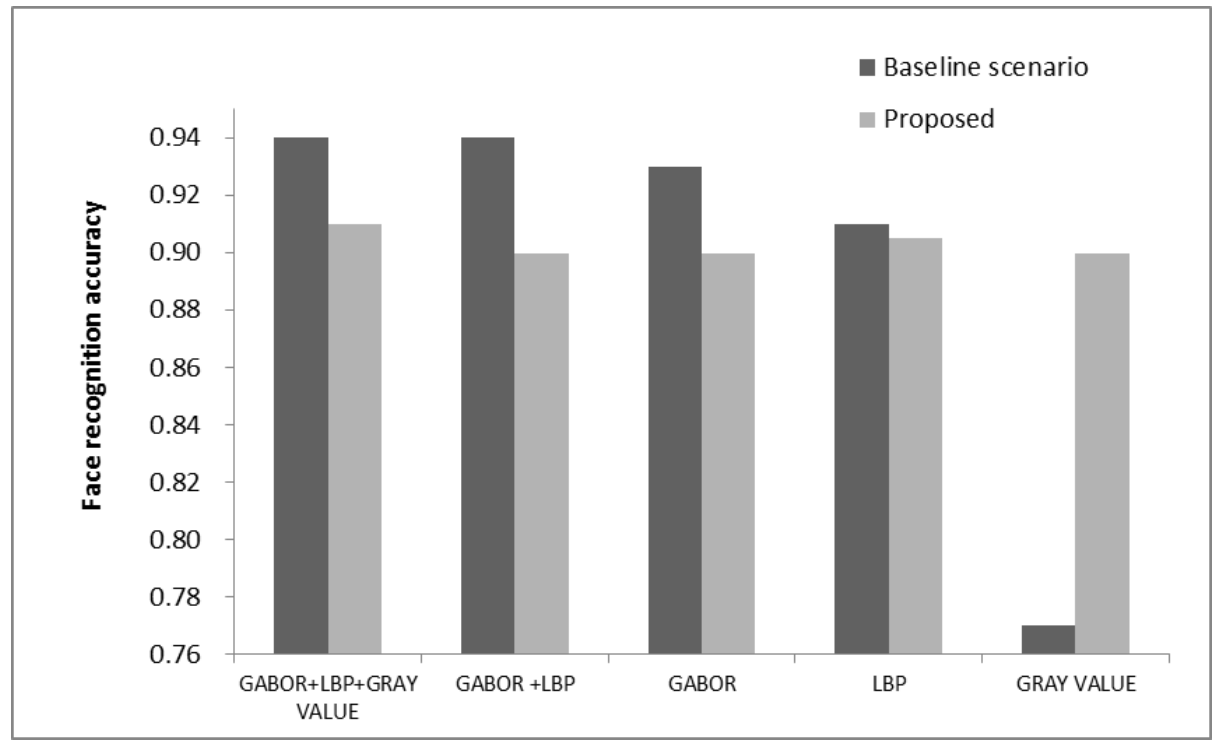

(b) Face recognition accuracy

Fig. 7. Comparison of performance between baseline and proposed approaches on various face feature extraction algoritms

\section{Conclusion}

In this paper, we proposed a method to improve the energy efficiency of a multicore-based handheld device and satisfy the required face recognition accuracy by implementing the optimal control parameters (i.e., CPU frequency, number of cores, and compression parameter) with respect to the given parameters (i.e., compression application parallelism, network bandwidth). First, the tradeoff between face recognition accuracy and energy consumption was analyzed with $E-A$ (i.e., Energy-Accuracy) analysis, and then, we find the optimal parameters from the $E-A$ analysis. Finally, we compared the compression and un_compression 
approaches, and improved energy efficiency while satisfying the minimum requirement for face recognition accuracy. In our experiments, we used a multicore-based handheld device (i.e., an Intel quad-core i7) to analyze the machine's characteristics, and we analyzed energy efficiency by measuring the actual power consumption with a WT210 tool. We also used the JPEG compression algorithm and diverse network conditions. Based on our experimental results, we prove that the proposed approach can enhance the energy efficiency of $G A B O R+L B P+G R A Y V A L U E, G A B O R+L B P, G A B O R$, and $L B P$ by factors of 6.8, 3.6, 3.6, and 2.4 over the baseline, respectively, while satisfying user's face recognition accuracy.

\section{Acknowledgments}

This work was supported by Basic Science Research Program through the National Research Foundation of Korea(NRF) funded by the Ministry of Science, ICT \& Future Planning(NRF-2015R1C1A1A02037688)

\section{References}

[1] https://fidoalliance.org/ (accessed on 2016).

[2] M. Sahani, S. Subudhi, and M. Mohanty, "Design of Face Recognition based Embedded Home Security System,” TIIS, vol. 10, no. 4, pp. 1751-1767, April, 2016. Article (CrossRef Link)

[3] Q. Lin, J. Yang, N. Ye, R. Wang, and B. Zhang, "Face Recognition in Mobile Wireless Sensor Networks," International Journal of Distributed Sensor Networks, vol. 9, no. 9, August, 2013. Article (CrossRef Link)

Y. Y. Park, Y. Choi, and K. Lee, "A Study on the Design and Implementation of Facial Recognition Application System,” International Journal of Bio-Science and Bio-Technology, vol. 6, no. 2, pp.1-10, April, 2014. Article (CrossRef Link)

[4] A. T. Tran, J. Y. Kim, A. Chaudhry, B. Pham, and H-. G. Kim, "Visual Observation Confidence based GMM Face Recognition robust to Illumination Impact in a Real-world Database," TIIS, vol. 10, no.4, April, 2016. Article (CrossRef Link)

[5] A. Gorea and S. Guptab, "Full reference image quality metrics for JPEG compressed images," AEU-International Journal of Electronics and Communications, vol. 69, no. 2, pp. 604-608, February, 2015. Article (CrossRef Link)

[6] A. M. Kishk, N. W. Messiha, N. A. El-Fishawy, A. A. Alkafs, and A. H. Madian, "Low Energy Lossless Image Compression Algorithm for Wireless Sensor Network (LE-LICA)," Sensors \& Transducers Journal, vol. 188, no. 5, pp. 102-106, May, 2015. Article (CrossRef Link)

[7] G. Weinhandel, H. Stogner, and A. Uhl, "Experimental study on lossless compression of biometric sample data," Proc. of Image and Signal Processing and Analysis, pp. 517-522, September, 2009. Article (CrossRef Link)

[8] A. Sepas-Moghaddam and M. Moin, "Face recognition in colour JPEG compressed domain," International Journal of Biometrics, vol. 6, no. 3, pp. 304-320, August, 2014.

Article (CrossRef LInk)

[9] M. Gerards, J. Hurink, and J. Kuper, "On the interplay between global DVFS and scheduling tasks with precedence constraints," IEEE Transactions on Computers, vol. 64, no. 6, pp. 1742-1754, June, 2015. Article (CrossRef Link)

[10] S. K. Saurav, G. Prasad, and M. Chauhan, "Adaptive Power Management for HPC applications," Green High Performance Computing (ICGHPC), 2016 2nd International Conference on, pp. 26-27, February, 2016. Article (CrossRef Link)

[11] Y. Chen, J. Mair, Z. Huang, D. Eyers, and H. Zhang, "A State-Based Energy/Performance Model for Parallel Applications on Multicore Computers," in Proc. of Parallel Processing Workshops (ICPPW), 2015 44th International Conference on, pp. 230-239, September, 2015.

Article (CrossRef Link) 
[12] S. Lee, H. Kim, Y. Chung, and D. Park, "Energy Efficient Image/Video Data Transmission on Commercial Multi-Core Processors,” Sensors, vol. 12, no. 11, pp. 14647-14670, November, 2012. Article (CrossRef Link)

[13] S. Lee, H. Kim, and Y. Chung, "Power-Time Tradeoff of Parallel Execution on Multi-core Platforms,” Mobile, Ubiquitous, and Intelligent Computing, vol. 274, pp.157-163, 2014. Article (CrossRef Link)

[14] N. Hirofumi, N. Naoya, and T. Katsuya, “WT210/WT230 Digital Power Meters,” Yokogawa TR 35, pp. 17-20, 2003. http://tmi.yokogawa.com/technical-library/white-papers/wt210wt230-digital-power-meters/

[15] http://cvc.yale.edu/projects/yalefaces/yalefaces.html (accessed on 2016).

[16] Z. He, Y. Liang, L. Chen, A. Hmad, and D. Wu, "Power-rate-distortion analysis for wireless video communication under energy constraints," IEEE Transactions on Circuits and Systems for Video Technology, vol. 15, no. 5, pp. 645-658, May, 2005. Article (CrossRef Link)

[17] Z. He, W. Cheng, and X. Chen, "Energy minimization of portable video communication devices based on power-rate-distortion optimization," IEEE Transactions on Circuits and Systems for Video Technology, vol. 18, no. 5, pp. 596-608, April, 2008. Article (CrossRef Link)

[18] D. Lee, H. Kim, M. Rahimi, D. Estrin, and J. Villasenor, "Energy-Efficient Image Compression for Resource-Constrained Platforms,” IEEE Transactions on Image Processing, vol. 18, no. 9, pp. 2100-2113, May, 2009. Article (CrossRef Link)

[19] S. Beak, B. Hieu, H. Lee, S. Choi, I. Kim, K. Lee, Y. Lee, and T. Jeong, "Novel binary tree Huffman decoding algorithm and field programmable gate array implementation for terrestrial-digital multimedia broadcasting mobile handheld," IET Science, Measurement and Technology, vol. 6, no. 6, pp. 527-532, November, 2012. Article (CrossRef Link)

[20] B. Barney, "POSIX Threads Programming,” Available online(accessed on 2016). http://www.llnl.gov/computing/tutorials/pthreads

[21] X. Tan and B. Triggs, "Fusing Gabor and LBP Feature Sets for Kernel-Based Face Recognition," International Workshop on Analysis and Modeling of Faces and Gestures, pp. 235-249, 2007. Article (CrossRef Link)

[22] L. A. Cament, F. J. Galdames, K. W. Bowyer, and C. A. Perez, "Face recognition under pose variation with local Gabor features enhanced by Active Shape and Statistical Models," Pattern Recognition, vol. 48, no. 11, pp. 3371-3384, November 2015. Article (CrossRef Link)

[23] D. Huang, C. Shan, M. Ardabilian, Y. Wang, and L. Chen, "Local Binary Patterns and Its Application to Facial Image Analysis: A Survey," IEEE Transactions on Systems, Man, and Cybernetics, Part C (Applications and Reviews), vol. 41, no. 6, pp. 765-781, March, 2011. Article (CrossRef Link)

[24] G. P. Nam, B. J. Kang, and K. R. Park, "Robustness of Face Recognition to Variations of Illumination on Mobile Devices Based on SVM,” TIIS, vol. 4, no.1, pp. 25-44, February, 2010. Article (CrossRef Link)

[25] X. Xiang, F. Liu, Y. Bi, Y. Wang, and J. Tang, "Local Similarity based Discriminant Analysis for Face Recognition,” TIIS, vol. 9, no.11, pp. 4502-4518, November, 2015. Article (CrossRef Link)

[26] W. Li and L. Wang, "Near-infrared Face Recognition by Fusion of E-GV-LBP and FKNN," TIIS, vol. 9, no.1, pp. 208-223, January, 2015. Article (CrossRef Link) 


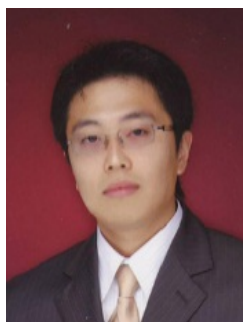

Sungju Lee received his BS and MS degrees from Korea University, Korea in 2006 and 2008, respectively. He also received $\mathrm{PhD}$ degree from Korea University in 2012. He had been a research professor at the Dept. of Computer Information, Korea University from 2012 to 2014. He is currently an assistance Professor in the Dept. of Computer Convergence Software, Korea University. His research interests include big data processing, information security, video encryption, and energy-efficiency of image compression.

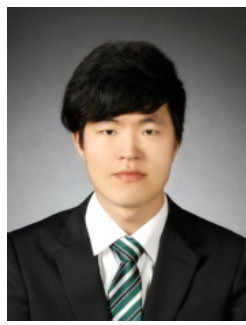

Jaewon Sa is currently a PhD student in the Dept. of Computer Convergence Software at Korea University. He received the BS and MS degrees in computer science from Korea University, Korea, in 2015 and 2017, respectively. His research interests include image processing, signal processing and parallel processing.

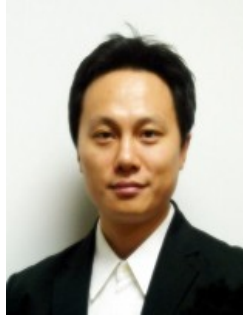

Hyeonjoong Cho is a professor in the Dept. of Computer and Information Science at Korea University, South Korea. He received PhD degree in computer engineering from Virginia Polytechnic Institute and State University (Virginia Tech) in 2006. He received MS degree in electronic and electrical engineering from the Pohang University of Science and Technology in 1998 and BS degree in electronic engineering from Kyungpook National University in 1996, South Korea. He has been a research staff member at Nanyang Technological University (NTU), Singapore, in 2015. Before he joined Korea University in 2009, he was a senior researcher at the Electronics and Telecommunications Research Institute, South Korea. Before pursuing the $\mathrm{PhD}$ degree, he was a senior software engineer at Samsung Electronics, South Korea. His research focuses on real-time systems, cyber-physical systems, ubiquitous computing, etc.

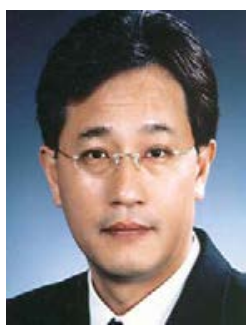

Daihee Park received his BS degree in mathematics from Korea University, Korea, in 1982, and his PhD degree in computer science from the Florida State University, USA, in 1992. He joined Korea University in 1993, where he is currently a professor in the Dept. of Computer Convergence Software. His research interests include data mining and intelligent database. 